\title{
Genes determining anthocyanin pigmentation in Solanum tuberosum L.
}

\author{
K.V. Strygina*, E.K. Khlestkina, A.V. Kochetov \\ Institute of Cytology and Genetics SB RAS, Novosibirsk, Russia \\ *e-mail: pushpandzhali@bionet.nsc.ru
}

Key words: gene duplication, flavonoids biosynthesis, MBW, Solanaceae, transcription factor

\begin{abstract}
Motivation and Aim: Anthocyanin pigments are plant secondary metabolites, having multiple biological functions in plants including potato Solanum tuberosum L. Activation of anthocyanin synthesis occurs with MBW complex, which is formed by transcription factors MYB, bHLH and WD40. Certain genes and their allelic variants affecting phenotype (purple or red tuber skin, purple or red tuber flash, purple leaves, stems) have been not yet identified. The aim of our research is to find allelic differences underlying phenotypic variation by coloration traits in potato and to develop allele-specific DNAmarkers for future markers-assisted potato breeding programs.
\end{abstract}

Methods and Algorithms: The search for sequences and genes structure identification was performed using the PGSC database. The search for functionally significant gene domains was carried out using InterPro. Development of diagnostic primers for the alleles determination as well as primers for the amplification of full-length transcripts of genes was performed using the OLIGO program. A total of $36 \mathrm{~S}$. tuberosum L. varieties and hybrids were studied. Eight of them, differring by anthocyanin pigmentation, were selected for further analysis of the expression of anthocyanin biosynthesis genes. Molecular cloning was performed using a pDrive cloning vector.

Results: The information about known MYB-, bHLH- and WD40-encoding genes was exploited to develop allele-specific markers for analysis of the regulatory genes. Diagnostic DNA markers were developed for each of the identified genes and their allelic variants. Using RT-PCR on eight genotypes differing by anthocyanin coloration we compared activity of certain $M Y B, b H L H$ and $W D 40$ gene copies. The regulatory genes of MYB (StAN1, StMYBA1, StMYB113), bHLH (StJAF13 and StbHLH1), WD40 (StWD40) families were isolated. We showed that the main regulator of anthocyanin coloration in leaves and stems among the MYB-encoding genes is StAN1. The expression of other MYB-encoding genes as well as that of the bHLH- and WD40- encoding genes did not correlate with the potato coloration.

Conclusion: We have developed intragenic markers, by which differences in StAN1 alleles can be detected. However, due to the high allelic diversity in this locus, the markers developed could not be used alone for prediction of potato stem and tuber anthocyanin coloration. To develop effective markers further analysis of nucleotide sequences in the promoter region and the identification of genetic polymorphisms association with the transcription level is required. Overall, the results of the study are important for understanding the mechanisms underlying tissue specific regulation of anthocyanin synthesis.

Acknowledgements: This study was supported by the Russian Science Foundation (No. 16-16-04073). 